\title{
A Survey of Low Visibility Image Enhancement Based on MSRCR Algorithm
}

\author{
Haokang Wen*, Hongbo Hao \\ Tianjin University of Science and Technology, China \\ E-mail: *haokangwen@foxmail.com \\ www.tust.edu.cn
}

\begin{abstract}
With the development of computer vision systems in the fields of traffic and safety monitoring, image enhancement has become an important research direction of computer vision. After development, the Retinex algorithm has a better enhancement effect, and the MSRCR algorithm has now developed into one of the important methods in image enhancement. This article introduces the development of the Retinex algorithm, and focuses on the main process and steps of the MSRCR algorithm, and uses the algorithm to enhance the low-visibility images of haze and night in multiple scenes. The actual results show that the MSRCR algorithm has a better enhancement effect and has a wide range of application values.
\end{abstract}

Keywords: image enhancement, MSRCR, Retinex algorithm

\section{Introduction}

Sometimes there will be some low visibility in daily life, such as the influence of haze caused by air pollution, and the situation of insufficient illumination at night, which will make the image acquired by the image acquisition system produce more obvious degradation phenomenon, such as low color saturation, poor definition of edge details, blurred image and so on. This will reduce the efficiency of later research and analysis. In order to improve the quality of image acquisition under low visibility and reduce the serious impact of fog weather on outdoor imaging system, many scholars and researchers have carried out a lot of research on image enhancement technology.

For the image defogging technology, the multi-scale information characteristics of the original image are usually taken as the main consideration factor, and the impurities in the non-sensitive area of the image are eliminated by compensating the contrast, brightness and color saturation of the image scene, so as to improve the visual effect of the image. At present, there are many algorithms for image enhancement, including global and local histogram equalization, wavelet transform enhancement and Retinex based on color constancy theory. This paper introduces the Retinex algorithm.

\section{Development of Retinex Algorithm}

\subsection{The basic model of Retinex}

Retinex is a commonly used image enhancement method based on scientific experiments and scientific analysis. It was proposed by Edwin H. land ${ }^{1}$. Retinex is a word made up of two words: retina and cortex. Land's Retinex model is based on three assumptions:

- The color we perceive is the result of the interaction between light and matter. The water we see is colorless, but the water film soap film is colorful, which is the result of light interference on the surface of the film.

- Each color region is composed of red, green and blue primary colors of a given wavelength.

- The primary colors determine the color of each unit area. 
The basic theory of Retinex theory is that the color of an object is determined by its ability to reflect long wave (red), medium wave (green) and short wave (blue) light. Color uniformity is the basis of color uniformity. Different from the traditional linear and nonlinear methods, Retinex can achieve a balance in dynamic range compression, edge enhancement and color constancy. Therefore, Retinex can enhance various types of images adaptively.

The main steps are as followed:

According to the image formation model, an image can be expressed as:

$$
\mathrm{I}(\mathrm{x}, \mathrm{y})=\mathrm{S}(\mathrm{x}, \mathrm{y}) \cdot \mathrm{R}(\mathrm{x}, \mathrm{y})
$$

$(\mathrm{x}, \mathrm{y})$ is the coordinates of the pixels in the image, " $\mathrm{S}$ " represents the incident light, " $R$ " represents the reflection characteristics of the object, "I" is the reflected light, which is captured by the camera as an image.

Take the logarithm to separate the incident light component $\mathrm{S}$ and the reflected light component $\mathrm{R}$. I'( $\mathrm{x}, \mathrm{y})$ is the logarithm of $\mathrm{I}(\mathrm{x}, \mathrm{y})$.

$$
\begin{aligned}
& I^{\prime}(\mathrm{x}, \mathrm{y})=\log [\mathrm{S}(\mathrm{x}, \mathrm{y}) \cdot \mathrm{R}(\mathrm{x}, \mathrm{y})] \\
& =\log (\mathrm{S}(\mathrm{x}, \mathrm{y}))+\log (\mathrm{R}(\mathrm{x}, \mathrm{y}))
\end{aligned}
$$

Convolve the original image with a Gaussian template, which is equivalent to low-pass filtering the original image to obtain a low-pass filtered image $\mathrm{D}(\mathrm{x}, \mathrm{y})$, where $\mathrm{F}(\mathrm{x}, \mathrm{y})$ represents Gaussian filtering function:

$$
\mathrm{D}(\mathrm{x}, \mathrm{y})=\mathrm{I}^{\prime}(\mathrm{x}, \mathrm{y}) \cdot \mathrm{F}(\mathrm{x}, \mathrm{y})
$$

Subtract the low-pass filtered image from the original image to obtain the high-frequency enhanced image $\mathrm{G}(\mathrm{x}, \mathrm{y})$.

$$
\mathrm{G}(\mathrm{x}, \mathrm{y})=\mathrm{I}^{\prime}(\mathrm{x}, \mathrm{y})-\mathrm{D}(\mathrm{x}, \mathrm{y})
$$

In the previous steps, the incident light component $\mathrm{S}$ and the reflected light component $\mathrm{R}$ are separated, so the antilog of the resultant high-frequency enhanced image $\mathrm{G}(\mathrm{x}, \mathrm{y})$ must be taken to obtain the enhanced image $\mathrm{R}(\mathrm{x}, \mathrm{y})$.

$$
\mathrm{R}(\mathrm{x}, \mathrm{y})=\exp (\mathrm{G}(\mathrm{x}, \mathrm{y}))
$$

The contrast enhancement of $\mathrm{R}(\mathrm{x}, \mathrm{y})$ is performed to obtain the final result image. As mentioned above, the flowchart of the Retinex algorithm is shown in Fig.1.

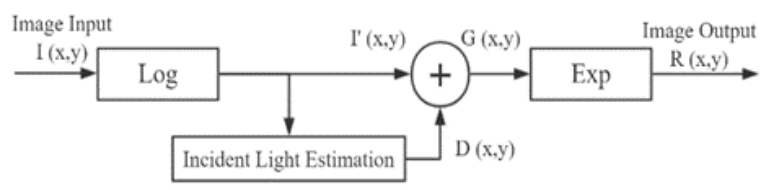

Fig.1 Flow chart of Retinex algorithm

\subsection{SSR algorithm}

Researchers such as Jobson proposed the single-scale Retinex (SSR) algorithm ${ }^{2,3}$. The specific formula is as follows:

$$
\mathrm{R}_{\mathrm{i}}(\mathrm{x}, \mathrm{y})=\log \mathrm{I}_{\mathrm{i}}(\mathrm{x}, \mathrm{y})-\log \left[\mathrm{I}_{\mathrm{i}}(\mathrm{x}, \mathrm{y}) * \mathrm{~F}(\mathrm{x}, \mathrm{y})\right]
$$

In the formula, $\mathrm{R}_{\mathrm{i}}(\mathrm{x}, \mathrm{y})$ is the output of Retinex in the " $\mathrm{i}$ " color spectrum, $\mathrm{I}_{\mathrm{i}}(\mathrm{x}, \mathrm{y})$ is the image distribution, that is, the brightness value at the position $(\mathrm{x}, \mathrm{y}) .{ }^{*}$ represents the convolution operation, $\mathrm{F}(\mathrm{x}, \mathrm{y})$ is a wraparound function that is defined by equation (7).

$$
\mathrm{F}(\mathrm{x}, \mathrm{y})=\mathrm{K} \cdot e^{\frac{-\left(x^{2}+y^{2}\right)}{\sigma^{2}}}
$$

Among them, $\sigma$ is the wrapping scale, $\mathrm{K}$ is the normalization constant. The wrapping function satisfies:

$$
\iint \mathrm{F}(\mathrm{x}, \mathrm{y}) \mathrm{dxdy}=1
$$

The stronger the dynamic compression capability of SSR is, the better the details of the dark part of the image can be enhanced, but the color distortion of the output image is more serious.

\subsection{MSR algorithm}

MSR is developed on the basis of SSR algorithm ${ }^{4}$. The specific formula of MSR is as followed:

$$
\begin{aligned}
& \mathrm{R}_{\mathrm{i}}(\mathrm{x}, \mathrm{y})=\sum_{\mathrm{k}=1}^{\mathrm{K}} \mathrm{w}_{\mathrm{k}}\left\{\log \mathrm{I}_{\mathrm{i}}(\mathrm{x}, \mathrm{y})-\log \left[\mathrm{I}_{\mathrm{i}}(\mathrm{x}, \mathrm{y}) * \mathrm{~F}(\mathrm{x}, \mathrm{y})\right]\right\}, \\
& \mathrm{i}=1, \ldots, \mathrm{N}
\end{aligned}
$$

Among them, i represents the i-th color channel, and $(\mathrm{x}, \mathrm{y})$ represents the coordinates of the pixel in the image. $\mathrm{N}$ is the number of color channels in the image. $\mathrm{N}=1$ represents a grayscale image, $\mathrm{N}=3$ represents a color image, $i \in(R, G, B)$. $I_{i}(x, y)$ represents the $i$-th color channel in the input image, $\mathrm{R}_{\mathrm{i}}(\mathrm{x}, \mathrm{y})$ represents the output result of the MSR of the i-th channel. $\mathrm{F}(\mathrm{x}, \mathrm{y})$ is a Gaussian function, $\mathrm{k}$ represents the number of Gaussian surround functions or the number of surround scales, $\mathrm{w}_{\mathrm{k}}$ represents the weight related to the Gaussian function, $\sum_{\mathrm{k}=1}^{\mathrm{K}} \mathrm{W}_{\mathrm{k}}=1$, in general, MSR takes three scales of high, medium and low, $\mathrm{K}=3$. As shown in the Fig. 2, it is the flow chart of MSR algorithm.

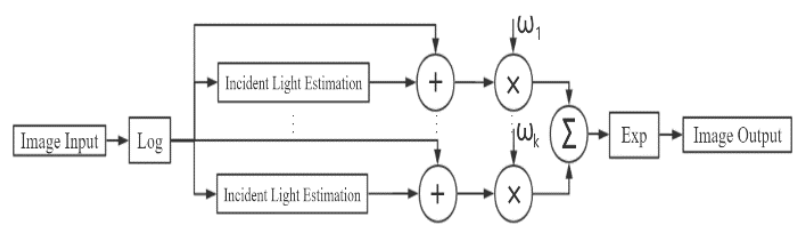

Fig.2 Flow chart of MSR algorithm

(C) The 2021 International Conference on Artificial Life and Robotics (ICAROB2021), January 21 to 24, 2021 


\subsection{Basic theory of MSRCR algorithm}

The algorithm with color recovery factor originated from Retinex theory, which adds a color recovery factor to the multi-scale algorithm ${ }^{5,6}$.

$$
\left\{\begin{array}{c}
\mathrm{R}_{\mathrm{i}}^{\mathrm{MSRCR}}(\mathrm{x}, \mathrm{y})=\mathrm{C}_{\mathrm{i}}(\mathrm{x}, \mathrm{y}) \mathrm{R}_{\mathrm{i}}^{\mathrm{MSR}}(\mathrm{x}, \mathrm{y}) \\
\mathrm{C}_{\mathrm{i}}(\mathrm{x}, \mathrm{y})=\beta \log \left[\alpha \mathrm{I}_{\mathrm{i}}^{\prime}(\mathrm{x}, \mathrm{y})\right] \\
\mathrm{I}_{\mathrm{i}}^{\prime}(\mathrm{x}, \mathrm{y})=\mathrm{I}_{\mathrm{i}}(\mathrm{x}, \mathrm{y}) / \sum_{\mathrm{i}=1}^{3} \mathrm{I}_{\mathrm{i}}(\mathrm{x}, \mathrm{y})
\end{array}\right.
$$

Among them, $i \in(R, G, B), C_{i}$ is the color recovery coefficient of the $\mathrm{i}$-th channel, which is the key factor, $\beta$ is the gain constant, $\alpha$ is the controlled nonlinear enhancement coefficient, $R_{i}^{M S R C R}$ is the output image of the $\mathrm{i}$-th channel processed by this algorithm.

The MSRCR algorithm is based on MSR algorithm, adding the adjustment factor $\mathrm{C}_{\mathrm{i}}$ which considers the ratio of $\mathrm{R}, \mathrm{G}$ and $\mathrm{B}$ in the original image. Because the color recovery factor has color compensation for the image enhanced by MSR algorithm, the image enhanced by MSRCR can better maintain the original color without distortion.

\section{Test Result}

The main purpose of image enhancement is to suppress the useless noise in the process of image acquisition and transmission, highlight the useful information in the image, make the image conform to the visual effect of human eyes as much as possible, or make the image transform into a form that is conducive to computer recognition and analysis, and improve the subsequent processing ability and application value of the image.

In this paper, under the Windows 10, based on MATLAB 2015a software, the MSRCR algorithm is used to process the fuzzy images in haze and night. as shown in the Table 1 below, it is the computer environment.

Table 1. Computer environment

\begin{tabular}{cc}
\hline GPU & NVIDIA GeForce MX150 \\
Video memory & $2 \mathrm{~GB}$ \\
Operating system & Windows 10 \\
Software version & MATLAB R2015a \\
\hline
\end{tabular}

As shown in the Fig. 3 below, the image on the left is the original image, and the image on the right is enhanced by this algorithm. The image in haze weather is processed, and the details of the enhanced image are more obvious. In particular, the color of the object in the original image is grayish white, but after enhancement, the color is more vivid.

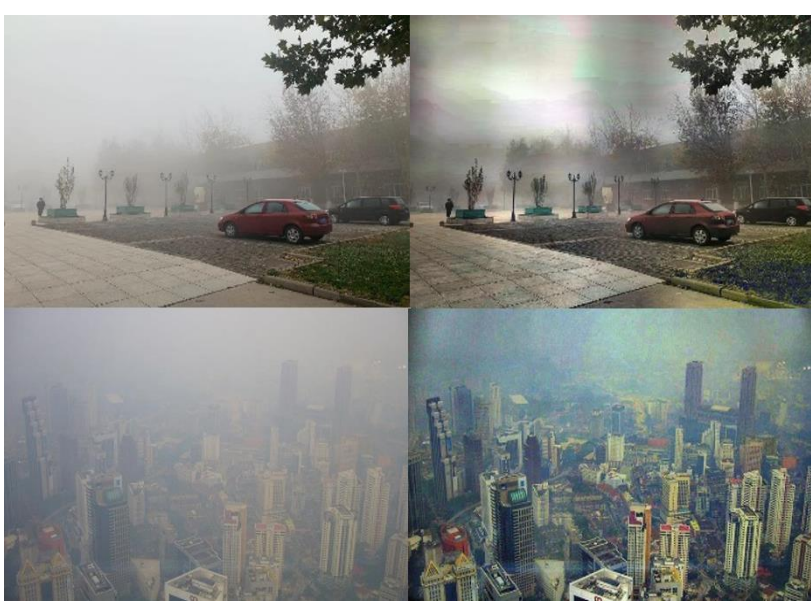

Fig.3 Comparison of haze image enhancement effect

As shown in the Fig.4 below, the edge outline and details of the object in the original image are very fuzzy and difficult to distinguish. The enhanced image on the right has better visual effect and clearer object contour after processing the image at night.

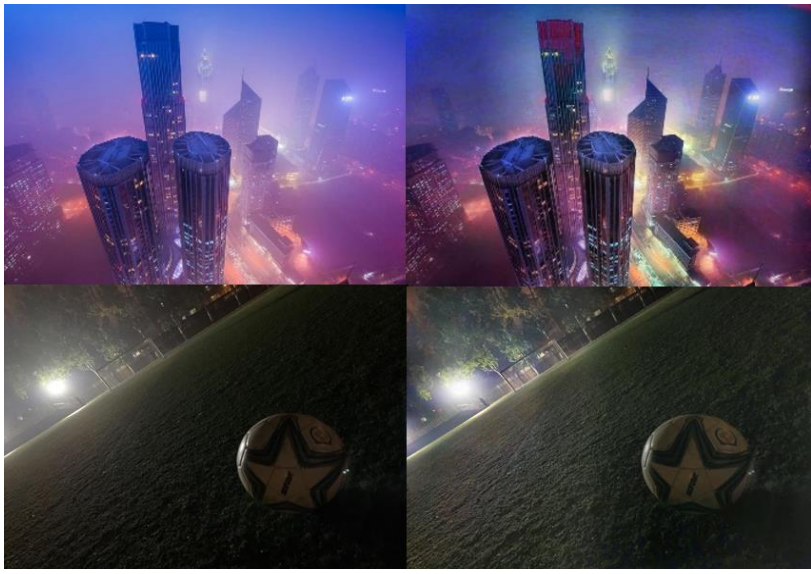

Fig.4 Comparison of night image enhancement effect

As shown in the two figures, it can be seen from the comparison that, although there are halos in some areas of the images, the overall enhancement effect is satisfactory due to the clearer details and brighter colors of the objects. 


\section{Conclusion}

The theory and development of Retinex algorithm are introduced in this paper. Especially the process and method of SSR, MSR, MSRCR algorithm are introduced. At the end of the article, the MSRCR algorithm is used to for practical test and the experimental results are remarkable and satisfied.

The test results show that the enhancement effect of this method is obvious and has certain practicability. Retinex image enhancement algorithm based on human visual system has the advantages of color constancy, image sharpening and high fidelity, which cannot be compared with traditional enhancement algorithm. The color information of the enhanced image can be restored or maintained well.

\section{References}

1. Land E H. The retinex theory of color vision. Scientific american, 1977, 237(6): pp. 108-129.

2. Jobson D J, Rahman Z, Woodell G A, "Properties and performance of a center/surround retinex," IEEE Transactions on Image Processing, 1997, 6(3): pp. 451-462.

3. Hines G, Rahman Z, Jobson D, et al. Single-scale retinex using digital signal processors. 2005.

4. Rahman Z, Jobson D J, Woodell G A. Multi-scale retinex for color image enhancement//Proceedings of 3rd IEEE International Conference on Image Processing. IEEE, 1996, 3: pp. 1003-1006.

5. Jobson D J, Rahman Z, Woodell G A. Retinex image processing: Improved fidelity to direct visual observation//Color and Imaging Conference. Society for Imaging Science and Technology, 1996, 1996(1): pp. 124-125.

6. Girija M G, Shanavaz K T, Ajith G S. Image dehazing using MSRCR algorithm and morphology based algorithm: A Concise Review. Materials Today: Proceedings, 2020, 24: pp. 1890-1897. 